\title{
CPAP or non-invasive ventilation in obesity hypoventilation syndrome: does it matter which one you start with?
}

\author{
Julio R Noda, ${ }^{1}$ Juan F Masa, ${ }^{2,3}$ Babak Mokhlesi ${ }^{1}$
}

In recent decades, the prevalence of obesity and severe obesity has increased significantly around the globe. ${ }^{12}$ As a consequence, it is likely that the prevalence of obesity-associated comorbidities such as obesity hypoventilation syndrome (OHS) will follow the same trend. ${ }^{3}$ OHS is the most severe form of respiratory compromise induced by obesity, leading to increased mortality and a wide array of comorbidities such as pulmonary hypertension, right heart failure and increased risk of hospitalisation due to acute-on-chronic hypercapnic respiratory failure. ${ }^{4-6}$ Unfortunately, OHS remains frequently unrecognised or misdiagnosed even in patients with severe obesity hospitalised with hypercapnic respiratory failure. ${ }^{78}$

Although positive airway pressure (PAP) remains the cornerstone therapy for OHS, controversy persists as to the preferred mode of PAP therapy for long-term management. ${ }^{9}$ In theory, non-invasive ventilation (NIV) should be more effective than CPAP since it addresses the various complex pathophysiological disturbances that result in OHS, such as altered ventilatory drive, increased work of breathing due to restrictive chest physiology induced by excess adiposity and exacerbation of hypoventilation during sleep. However, observational and a few randomised controlled trials with short-term follow-up have shown that both CPAP and NIV are equally effective in improving daytime and nighttime hypercapnia as well as symptoms in patients with OHS. ${ }^{10-12}$ NIV is commonly prescribed as a fixed level of pressure support in the form of bilevel PAP in

\footnotetext{
${ }^{1}$ Department of Medicine, Section of Pulmonary and Critical Care, Sleep Disorders Center, The University of Chicago, Chicago, Illinois, USA; ${ }^{2}$ San Pedro de Alcántara Hospital, Cáceres, Spain; ${ }^{3}$ Centro de Investigación Biomédica en Red de Enfermedades Respiratorias (CIBERES), Madrid, Spain

Correspondence to Professor Babak Mokhlesi, Department of Medicine, Section of Pulmonary and Critical Care, Sleep Disorders Center, The University of Chicago, 5841 S. Maryland Ave, MC6076/Room M630, Chicago, IL 60637-1470, USA
}

bmokhles@medicine.bsd.uchicago.edu spontaneous mode or bilevel PAP spontaneous-timed (ST) mode with a back-up respiratory rate, or a variable level of pressure support such as volumetargeted pressure support. Given the lack of consensus and the limited information on long-term outcomes with the various modalities of PAP therapy, it is not surprising that there is significant variation in clinical practice.

To further address this relevant clinical question, Howard et $a l^{13}$ conducted a multicentre, randomised, parallel group, double-blind clinical trial comparing bilevel PAP ST $(n=29)$ with CPAP $(n=31)$ over 3 months in patients with newly diagnosed OHS recruited from ventilatory failure services at three Australian centres. The mean age of the participants was $53 \pm 10$ years, $53 \%$ were women, the mean body mass index was $55 \pm 11.9 \mathrm{~kg} / \mathrm{m}^{2}$ and the mean partial pressure of $\mathrm{CO}_{2}$ in arterial blood $\left(\mathrm{PaCO}_{2}\right)$ was $59.6 \pm 13.8 \mathrm{~mm} \mathrm{Hg}$ on initial presentation. At baseline, there were no significant differences between the two groups. Polysomnography was available in 47 of these patients with a mean apnoeahypopnoea index of $82 \pm 45$ events/hour, consistent with severe obstructive sleep apnoea (OSA). In the bilevel PAP ST group, the mean inspiratory pressure was $19.3 \mathrm{~cm} \mathrm{H}_{2} \mathrm{O}$ and the mean expiratory pressure was $11.9 \mathrm{~cm} \mathrm{H}_{2} \mathrm{O}$ with a back-up respiratory rate of 15 breaths $/ \mathrm{min}$. In the CPAP group, the mean pressure was $15.2 \mathrm{~cm} \mathrm{H}_{2} \mathrm{O}$.

The composite primary end point of hospital admission, persistence or worsening of ventilatory failure (defined as failure of $\mathrm{PaCO}_{2}$ to fall below $60 \mathrm{~mm} \mathrm{Hg}$ within 3 months or a rise in $10 \mathrm{~mm} \mathrm{Hg}$ from baseline) or non-adherence (defined as average PAP use of $<2$ hours/night) did not differ among the two groups (bilevel PAP ST $14.8 \%$ vs CPAP $13.3 \%, \mathrm{p}=0.87$ ). Of note, these outcomes were mostly driven by hospital admissions and nonadherence to PAP therapy, not persistent ventilatory failure. Similarly, improvements in $\mathrm{PaCO}_{2}, \mathrm{PaO}_{2}$ and serum bicarbonate were not significantly different between the two PAP modalities.
Secondary end points such as sleepiness, health-related quality of life (HRQoL), weight, physical activity and maximal inspiratory pressure improved with both PAP modalities, without group differences. Lastly, after exploratory analysis a significant correlation was found between baseline $\mathrm{PaCO}_{2}$ and persistence of ventilatory failure at 3 months (OR 2.3, p=0.03). The investigators concluded that initiating treatment with either bilevel PAP ST or CPAP in patients with newly diagnosed OHS was equally effective in preventing hospitalisation, controlling respiratory failure and improving HRQoL.

This clinical trial is a welcome addition to the limited number of trials comparing effectiveness between CPAP and NIV in OHS. One of the strengths of the study is that enrolment criteria were not as restrictive compared with other trials, thereby increasing the generalisability of their findings and rendering it more reflective of 'real-life' clinical practice. Although the degree of OSA in this study is in keeping with the severity of OSA in previous trials of OHS, ${ }^{11} 12$ their patients were more obese and had worse pulmonary function impairment. Nearly $42 \%$ of the patients $(n=25)$ were identified during an episode of hospitalisation due to acute-on-chronic hypercapnic respiratory failure; 21 of them were treated with bilevel PAP therapy prior to randomisation in order to achieve a stable arterial $\mathrm{pH}$ of 7.35-7.45. It is important to point out that NIV is the treatment of choice in hospitalised patients with OHS who are experiencing an acute-on-chronic hypercapnic respiratory failure. Once the acute component of hypercapnic respiratory failure was treated, 11 patients were randomised to bilevel PAP ST and 14 were randomised to CPAP. Therefore, it is not surprising that the mean baseline $\mathrm{PaCO}_{2}$ at the time of randomisation had decreased to $\sim 50 \mathrm{~mm} \mathrm{Hg}$ in the bilevel PAP ST group and $52.5 \mathrm{~mm} \mathrm{Hg}$ in the CPAP group (see figure 3 in the article by Howard et al.), levels that are similar to prior randomised controlled trials. ${ }^{11} 12 \quad 14$ Another important distinction, compared with more recent trials, is the lower level of pressure support used in the present study; levels that were similar to those used in a trial of patients with milder OHS. ${ }^{15}$ The mean pressure support (ie, difference between inspiratory and expiratory pressures in the bilevel PAP ST group) was $\sim 7.4 \mathrm{~cm} \mathrm{H}_{2} \mathrm{O}$ in the study by Howard et al as opposed to $\sim 12.2 \mathrm{~cm} \mathrm{H}_{2} \mathrm{O}$ in the study by Masa et $\mathrm{al}^{12}$ (mean inspiratory pressure of $20 \mathrm{~cm} \mathrm{H}_{2} \mathrm{O}$ and mean expiratory pressure 
of $7.8 \mathrm{~cm} \mathrm{H}_{2} \mathrm{O}$ with a backup rate of 14 breaths/min) and $\sim 13 \mathrm{~cm} \mathrm{H}_{2} \mathrm{O}$ in the study by Murphy et al ${ }^{14}$ (mean inspiratory pressure of $23 \mathrm{~cm} \mathrm{H}_{2} \mathrm{O}$ and mean expiratory pressure of $10 \mathrm{~cm} \mathrm{H}_{2} \mathrm{O}$ with a backup respiratory rate of 14 breaths/ min). This is in part related to the PAP titration protocol followed by Howard et al, where the expiratory pressure was increased until all obstructive respiratory events (apnoeas, hypopnoeas, flow limitation and snoring) were resolved in contrast to the other two studies in which expiratory pressure was increased only to resolve obstructive apnoeas. Whether a lower level of pressure support led to suboptimal control of nocturnal hypoventilation remains unknown. It is important to point out that despite the larger levels of pressure support provided in the study by Masa et al, ${ }^{12}$ CPAP and NIV were comparable in improving $\mathrm{PaCO}_{2}$. However, NIV was superior to CPAP in improving 6 min walk distance, $\mathrm{FEV}_{1}$ and certain aspects of HRQoL. It is certainly plausible that higher levels of pressure support would have been more beneficial in the cohort enrolled by Howard et al given that they were more obese with more significant lung function impairment. Interestingly, adherence to CPAP or NIV therapy in several trials of OHS, including the one by Howard et al, ${ }^{13}$ has been quite consistently between 5 and 6 hours/ night. $^{11} 12 \quad 14-16$

It is important to consider that the enrolled patients had clinically significant OSA and therefore the findings may not be extrapolated to patients with OHS with mild or no OSA. In these patients, CPAP may not be effective. Although NIV has been shown to be superior to lifestyle changes in patients with OHS without severe OSA, ${ }^{16}$ to our knowledge there is no randomised trial comparing CPAP with bilevel PAP in this particular phenotype of OHS. While data from several observational studies suggest that longterm NIV is associated with better survival rates, ${ }^{8}$ the Pickwick study by the Spanish Sleep Network-the largest trial of OHS with 36 months of follow-up-will shed further light on whether long-term NIV is more effective than CPAP therapy. ${ }^{12} 17$

While we eagerly await the results of the long-term Pickwick study, Howard et al have made an incremental and significant contribution to the current available evidence supporting the notion that clinically stable patients with OHS can be effectively managed with CPAP or bilevel PAP ST in the short-term. We cannot stress any further the importance of close clinical follow-up once PAP therapy is initiated to ensure adequate adherence and response to therapy, ${ }^{10}$ particularly since poor adherence to PAP therapy has been associated with increased mortality. ${ }^{6}$ Finally, successful management of OHS should consist of a multidisciplinary approach in order to effectively address the various facets of this complex condition including obesity, physical inactivity and management of cardiometabolic comorbidities. $^{18}$

Contributors All authors participated in drafting the editorial.

Competing interests JRN and JFM have no conflicts of interest to declare. BM is supported by National Institutes of Health grant R01HL119161 and has served as a consultant to Philips/Respironics and has received research support from Philips/Respironics. He has also received honorarium from Zephyr Medical Technologies and has served on the advisory board of Itamar Medical.

Provenance and peer review Commissioned; externally peer reviewed.

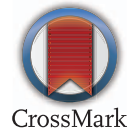

To cite Noda JR, Masa JF, Mokhlesi B. Thorax 2017; 72:398-399.

Published Online First 27 January 2017

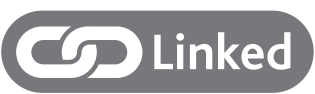

http://dx.doi.org/10.1136/thoraxjnl-2016-208559

Thorax 2017:72:398-399

doi:10.1136/thoraxjnl-2016-209607

\section{REFERENCES}

1 Ogden $\mathrm{CL}$, Carroll MD, Flegal KM. Prevalence of obesity in the United States. JAMA 2014:312:189-90.

$2 \mathrm{Ng} \mathrm{M}$, Fleming T, Robinson M, et al. Global, regional, and national prevalence of overweight and obesity in children and adults during 19802013: a systematic analysis for the Global Burden of Disease Study 2013. Lancet 2014;384: 766-81.
3 Balachandran JS, Masa JF, Mokhlesi B. Obesity hypoventilation syndrome epidemiology and diagnosis. Sleep Med Clin 2014;9:341-7.

4 Carrillo A, Ferrer M, Gonzalez-Diaz G, et al. Noninvasive ventilation in acute hypercapnic respiratory failure caused by obesity hypoventilation syndrome and chronic obstructive pulmonary disease. Am J Respir Crit Care Med 2012;186:1279-85.

5 Nowbar S, Burkart KM, Gonzales R, et al. Obesity-associated hypoventilation in hospitalized patients: prevalence, effects, and outcome. Am J Med 2004;116:1-7.

6 Castro-Añón O, Pérez de Llano LA, De la Fuente Sánchez $\mathrm{S}$, et al. Obesity-hypoventilation syndrome: increased risk of death over sleep apnea syndrome. PLOS ONE 2015;10:e0117808.

7 Marik PE, Chen C. The clinical characteristics and hospital and post-hospital survival of patients with the obesity hypoventilation syndrome: analysis of a large cohort. Obes Sci Pract 2016;2:40-7.

8 Pépin JL, Borel JC, Janssens JP. Obesity hypoventilation syndrome: an underdiagnosed and undertreated condition. Am I Respir Crit Care Med 2012:186:1205-7.

9 Mokhlesi B. Positive airway pressure titration in obesity hypoventilation syndrome: continuous positive airway pressure or bilevel positive airway pressure. Chest 2007:131:1624-6.

10 Mokhlesi B, Tulaimat A, Evans AT, et al. Impact of adherence with positive airway pressure therapy on hypercapnia in obstructive sleep apnea. I Clin Sleep Med 2006:2:57-62.

11 Piper AJ, Wang D, Yee BJ, et al. Randomised trial of CPAP vs bilevel support in the treatment of obesity hypoventilation syndrome without severe nocturnal desaturation. Thorax 2008;63:395-401.

12 Masa JF, Corral J, Alonso ML, et al. Efficacy of different treatment alternatives for obesity hypoventilation syndrome. Pickwick study. Am J Respir Crit Care Med 2015;192:86-95.

13 Howard ME, Piper AJ, Stevens B, et al. A randomised controlled trial of CPAP versus non-invasive ventilation for initial treatment of obesity hypoventilation syndrome. Thorax 2017;72:437-44.

14 Murphy PB, Davidson C, Hind MD, et al. Volume targeted versus pressure support non-invasive ventilation in patients with super obesity and chronic respiratory failure: a randomised controlled trial. Thorax 2012;67(8):727-734.

15 Borel JC, Tamisier R, Gonzalez-Bermejo J, et al. Noninvasive ventilation in mild obesity hypoventilation syndrome: a randomized controlled trial. Chest 2012;141:692-702.

16 Masa JF, Corral J, Caballero C, et al. Non-invasive ventilation in obesity hypoventilation syndrome without severe obstructive sleep apnoea. Thorax 2016;71:899-906.

17 López-Jiménez MJ, Masa JF, Corral J, et al., Grupo cooperativo. Mid- and long-term efficacy of non-invasive ventilation in obesity hypoventilation syndrome: the Pickwick's study. Arch Bronconeumol 2016;52:158-65.

18 Borel JC, Borel AL, Monneret D, et al. Obesity hypoventilation syndrome: from sleep-disordered breathing to systemic comorbidities and the need to offer combined treatment strategies. Respirology 2012;17:601-10. 